\title{
The U.S. Regulations and the Protection of Pediatric Subjects
}

\section{Stephen Napier}

Assistant Professor of Philosophy, Villanova University, 800 Lancaster Ave, Villanova, PA 19085, USA

The United States has recently taken on the project of reviewing its regulations governing human subjects in research. ${ }^{1}$ There are a number of regulations governing research on human subjects, but I focus on two in particular, namely, the Code of Federal Regulations Title 21 Part 50, and the Department of Health and Human Services (DHHS) Code of Federal Regulations 45 Part 46. Both regulations include a sub-part (i.e., sub-part D) addressing research on children. The aim of this paper is to highlight an ethical lacuna according to which, the main category under which most pediatric research is approved does not offer any further protection for pediatric subjects compared to that afforded adult subjects. And since children often do not have the capacity to understand the risks and purpose of the research for which they participate, we come close to violating their dignity with such lax criteria.

The regulations under consideration here were developed in order to ensure that all research was conducted in a way that respected the dignity of human subjects. The chief motivating factors for such regulations were major ethical errors in the history of human subjects research (examples include the Nazi experiments, Tuskeegee (deliberate untreated syphilis), Willowbrook (deliberate inoculation of children with hepatitis), or Jewish Chronic Disease Hospital (deliberate inoculation of live cancer cells)). Without governmental oversight and articulation of ethical standards, researchers were left to their own consciences and in many cases, those consciences perceived only the scientific prospects of the study and not the dignity of the human subjects being used. ${ }^{2}$

Though the regulations were primarily developed to ensure the ethical conduct of research on adult human subjects, as noted above, there were "sub-parts" devoted specifically to vulnerable populations, e.g., children, pregnant women/fetuses, and prisoners. ${ }^{4}$ The motivation behind these further sub-parts is that such subjects are compromised with respect to informed consent. Richard Behrman notes presciently, "because of the inherent vulnerabilities arising from their immaturity, infants, children, and adolescents need additional protections beyond what is provided to competent adults when they participate in research". ${ }^{5}$ Typically, we think of research as being just that; research

1. There are now a series of meetings, in particular Meeting Five, being conducted by the President's Council on Bioethics aimed to address this issue. See, http:// bioethics.gov/cms/meeting-five.

2. This explanation for the above mentioned errors does not cover the Nazi atrocities which possibly evade any explanation.

3. Vulnerable populations are ones for whom informed consent is severely compromised through developmental immaturity (fetus, children), degenerative disease (mentally disabled/Alzheimer's), or through environmental factors which may be coercive (prisoners).

4. A new subpart and/or Guidance document is being considered for adult subjects who do not have decision making capacity. In this category would be advanced Alzheimer's patients, or the mentally disabled/mentally ill. See the Secretary Advisory Committee on Human Research protections, meetings 0309, and 10-08, available at, http://www.hhs.gov/ohrp/sachrp/mtgings/mtg03-09/ present.html and http://www.hhs.gov/ohrp/sachrp/mtgings/mtg10-08/present. html respectively, (accessed, September 12, 2011).

5. Richard E. Behrman, "Preface," Ethical Conduct of Clinical Research Involving Children (Washington D.C.: National Academies Press, 2004).
- it is investigational. Investigators are finding out whether a drug, device, or procedure will be therapeutic. Thus, the chief end of research is knowledge not therapy. And insofar as the end is to gain knowledge and not necessarily to benefit the subject, at least not the subjects involved in the research, research necessarily requires using humans as a means to the end of knowledge. Ethically, this is clearly suspect, unless of course, the human subject shares the same end. And this is the ethical weight that informed consent must bear in the context of research. But this poses a problem for vulnerable populations involved in research: If vulnerable subjects have compromised decision-making ability and thus cannot give fully informed consent, and research is justifiable only if the subjects give informed consent, then research on vulnerable populations appears categorically unethical.

How, then, may one justify involving children or any vulnerable population, defined as an inability to give morally sufficient informed consent, in research? The answer has been, and still is, to look at the risk-benefit profile of the studies under consideration. If the risks are not that great, then, intuitively, the requirement to get full-fledged informed consent attenuates, and surrogate consent is sufficient. And this intuition is reflected in the DHHS regulations Sub-part D. The regulations attempt to give us a matrix of risk-benefit categories which, if the research is to be permissible, must fall within such categories. The first category of approvable research for children is research presenting no more than minimal risk to the child. For our purposes, this much is straightforward and presents no ethical difficulty. The next category, however, does present some worries. The following quotation is from section 405 of CFR 45 Part 46.

\section{$\$ 46.405$ Research involving greater than minimal risk but presenting the prospect of direct benefit to the individual subjects}

HHS will conduct or fund research in which the IRB finds that more than minimal risk to children is presented by an intervention or procedure that holds out the prospect of direct benefit for the individual subject, or by a monitoring procedure that is likely to contribute to the subject's well-being, only if the IRB finds that:

(a) The risk is justified by the anticipated benefit to the subjects;

(b) The relation of the anticipated benefit to the risk is at least as favorable to the subjects as that presented by available alternative approaches; and

Corresponding author: Stephen Napier, Assistant Professor of Philosophy, Villanova University, 800 Lancaster Ave, Villanova, PA 19085, USA, E-mail: Stephen.napier@villanova.edu

Received August 18, 2011; Accepted October 27, 2011; Published October 29 2011

Citation: Napier S (2011) The U.S. Regulations and the Protection of Pediatric Subjects. J Clinic Res Bioeth S2:001. doi:10.4172/2155-9627.S2-001

Copyright: (c) 2011 Napier S. This is an open-access article distributed under the terms of the Creative Commons Attribution License, which permits unrestricted use, distribution, and reproduction in any medium, provided the original author and source are credited. 
(c) Adequate provisions are made for soliciting the assent of the children and permission of their parents or guardians, as set forth in $\$ 46.408 .^{6}$

Four features of this category are worth noting. First, the risks of the research may be greater than minimal, but it does not stipulate how much greater. Research that may be extremely risky could be approvable within this category so long as the benefits "justify" the risk presented to the subjects. Second, in clause (a) the benefits that so justify the risks need only be "anticipated". Thus, the research may present known and well-established risks to the subjects, and yet be justified merely by the anticipated benefits. An illustrative example of balancing known risks with anticipated benefits is provided by Dyson and Parsi They note, An example of this application is Phase I oncology trials. (Phase I trials evaluate only safety, not efficacy.) While clearly the risks in a Phase I oncology trial are much greater than minor, such trials are most frequently approved under this category [46.405] with the justification that the potential benefit, when standard therapy offers little or no hope, is great ${ }^{7}$.

It is important to observe that Phase I oncology trials are noted here as examples of balancing known risks with anticipated benefits. But Phase I trials aim to analyze only safety. If Phase I studies are approved because of anticipated benefits as Dyson and Parsi purport here, then the term "anticipated" is being used very weakly. It is also notable that at least for category 405 , the anticipated benefits must accrue to the subjects in the study and not merely the subject-class (any and all patients with the same disease or condition). I note this not to call into question the ethical legitimacy of category 405 or Dyson and Parsi's application of it, but to fix our understanding of what "anticipated benefit" means. I happen to think that many Phase I trials in children are permissible, as do many others, but we need to be aware of the tenuous quality of our justifications for such studies.

These first two points, however, can be addressed by the specific activities of the Institutional Review Board (IRB) reviewing the study. In my own experience with IRB's, they typically do not permit studies where the risks are known, and grave, but the benefits are merely anticipated - applying that term very weakly as described above. Rather, many of the high-risk interventions being tested also show considerable promise in animal or adult studies. Although Phase I trials technically aim only for safety, there still is an expectation of efficacy nonetheless. (The reason for initiating a trial to begin with is not because one merely thinks that the drug/device/procedure is safe, but that it will also work. The investment required to conduct trials disposes researchers/ sponsors to study only those items that show considerable promise of 'going to market'.) The first note can be addressed by pointing out that the benefit in many cases of high-risk interventions is saving the life of a child. So, although I think the regulations as stated here logically permit studies which would strike one as clearly impermissible, an
IRB's application of these regulations is stricter. The criticism I do wish to focus on here is not that category 405 is deficient in itself, but compared to ethical criteria for adult studies, this category provides no further protection for pediatric subjects than that afforded adult subjects. And this conclusion, I believe, is important to take note of.

\section{Direct and anticipated benefit}

A fourth observation pertains to the meanings of the key terms involved in applying 405; terms such as "direct benefit," "anticipated benefit," "risks," and what counts as being "justified in relation to". Because significant work has already been done in this area, I will proceed here summarily.

A research project does not satisfy the contours of category 405 until it presents more than minimal risk, and a prospect of direct benefit. It is interesting to note, that there being a prospect of direct benefit does not alone justify the study, but it is permissible only if at least two other risk-related conditions are satisfied (i.e., (a), and (b)). Following closely here Nancy King ${ }^{8}$ and Field and Behrman ${ }^{9}$ I take the term "direct" as fundamentally a causal notion, and "benefit" as referring to specific types of benefits, as opposed to degree of magnitude. A direct benefit is a benefit "arising from receiving the intervention being studied" 10 . Typically, this benefit will be related to the health of the subject in question. That is, suppose the risks the intervention presents to subjects are harm to one's health via the toxic effects of the test drug. If the benefits are caused by the very intervention, i.e., administering the test article, then the benefits must be of the same sort as the risks.

Considering "direct benefit" as a causal notion is not only conceptually sensible, it satisfies important ethical criteria as well. Those working in research ethics typically eschew the idea of "extra" benefits compensating for risks of harm due to the research intervention. Friedman et al. quote favorably on this point from the Kenyan guidelines on the ethical conduct of research,

Extraneous benefits such as payment, or adjunctive medical services, such as the possibility of receiving a hepatitis vaccine not related to the research, cannot be considered in delineating the benefits compared with the risks, otherwise simply increasing payment or adding more unrelated services could make the benefits outweigh even the riskiest research ${ }^{11}$.

That is to say, there is a proportionality restriction on what types of benefits can ethically justify a study. More precisely, Proportionality restriction $=\mathrm{df}$ for any risk $\mathrm{R}$, and benefit $\mathrm{B}$, a research intervention is justified only if $\mathrm{R}$ is offset by $\mathrm{B}$, and $\mathrm{B}$ is of the same sort as $\mathrm{R}$.

The proportionality restriction satisfies two ethical criteria, one being that the benefits must be of the same sort - one cannot justify a study by simply paying subjects more money - and that for any risk R, there must be a compensating or offsetting benefit. The latter criterion

6. Department of Health and Human Services. Code of Federal Regulations. Title 45, Part 46, Protection of Human Subjects, subpart D Available at: http://www.hhs.gov/ ohrp/humansubjects/guidance/45cfr46.html [accessed September 21, 2011].

7. Dyson M, Parsi K 2010 "Characterizing the Level of Risk in Pediatric Research: An Ethical Examination of the Federal Regulations," Journal of Clinical Ethics 21: 212220 .

8. King NM 2000 "Defining and Describing Benefit Appropriately in Clinical Trials," J Law Med Ethics 28: 332-343.

9. Richard E. Behrman et al. note that "[a] direct benefit is a tangible positive outcome (e.g., cure of disease, relief of pain, and increased mobility) that may be experienced by an individual." Ethical Conduct of Clinical Research Involving Children p. 132.

10. King NM, "Defining and Describing Benefit Appropriately in Clinical Trials," p. 333.

11. Quoted from Alexander Friedman, Emily Robbins and David Wendler, "Which Benefits of Research Participation Count as 'Direct'?” Bioethics doi:10.1111/j.14678519.2010.01825.x, p. 3. Friedman et al. quoted from National Council for Science and Technology. 2004. Guidelines for Ethical Conduct of Biomedical Research Involving Human Subjects in Kenya. Nairobi: National Council for Science and Technology: 6. 
follows from work done on component analysis ${ }^{12}$. Field and Behrman explain, "When some [research] procedures present the prospect of direct benefit and others do not, the potential benefits from one component of the research should not be held to offset or justify the risks presented by another"13. To illustrate the point, Friedman et al. have us consider a study that holds out considerable promise of benefit to subjects with relatively low risks. Overall, the risk-benefit ratio clearly favors the benefits. Friedman et al. note,

If the ethics review committee were to evaluate the overall risks and benefits of the entire study only, the investigator might be able to add in a purely research biopsy that is of little value and justify its inclusion on the grounds that the potential benefits of the experimental medication are sufficient to justify its own risks, as well as the risks of the added biopsy. Component analysis is intended to block this potential for exploitation $^{14}$.

What is important for my purposes here is not just to be clear about the concepts employed in category 405 , but to note that the ethical reasons for 405 actually reach beyond research on children. Consider again the proportionality restriction. One may question why it is ethically necessary. By way of explaining its ethical necessity, consider an analogy with work done in the philosophy of religion. Philosophers of religion have long discussed the problem of evil according to which if there is a good and loving God, then why is there evil. Both theists and atheists alike agree that a successful answer to this question must obey important restrictions. Two are notable here: the suffering must be outweighed by or be defeated by a benefit (sometimes called a greater good), and that this benefit must accrue primarily to the sufferer ${ }^{15}$. The second restriction is especially pertinent in that a good and loving God would not use someone for the benefit or greater good of another. Furthermore, if the sufferer is suffering, for example, desolation or loneliness, the greater good must serve to resolve this desolation by, say, bringing about in the sufferer a greater sense of communion with others or with God. To the extent that the sufferer continues to suffer desolation or loneliness, even in the setting of other goods accruing to the sufferer, the suffering of desolation remains and is thereby an uncompensated evil. I would think that none of us would question this restriction. Part of the reason for its plausibility is that most would agree that a good and loving God would not in any way use people, or inflict suffering of some type and instance without compensating for that specific type and instance of suffering.

I wish to argue that our intuitions on what is a satisfactory answer to the problem of evil apply analogically to the research context and in particular, they support the proportionality restriction. Research risks are like the experience of suffering, and just as we would charge a good and loving God with using people if specific instances of suffering were left uncompensated, it appears we would do the same for a researcher who fails to compensate for each instance and type of risk. The important point here is not to focus on the analogs God and researcher, but to focus on the ethical reasons why we think God would be using the sufferer for the benefit of others, or why we think that God would fail to act justly by not compensating for each instance and type of suffering. Focusing on our ethical reasons in such a case, it seems the researcher must meet the same moral standards vis-à-vis compensation for both instances and types of risks in research to avoid the charge of merely using people or failing to act justly towards them.

This brief excursion into theodicy has a point: the proportionality restriction seems to apply to all research, not just pediatric research, in that it is a principle prohibiting the mere use of persons for the good of others. If this is correct, we can understand why justifying the risks in relation to the benefits is an independent ethical criterion governing the permissibility of a research project ${ }^{16}$. Even if subjects consent to a study whereby they would be treated merely as a means or otherwise unjustly, still renders the research impermissible. This is important in regard to my thesis that the regulations governing pediatric research do not afford greater protection than that afforded competent adults. My point so far is that the same basic ethical principle, i.e., the proportionality restriction, applies to both types of research.

So direct benefit is to be understood primarily as a causal notion whereby the research intervention causes, or holds out the prospect of causing, a benefit to the subject. But as noted above, this alone is not sufficient to justify a study. Let's consider condition (a) according to which the risks are justified in relation to the anticipated benefits. This is a separate and independent risk-related condition for two reasons: if anticipated benefits are to be identified with the direct benefits mentioned in the antecedent of 405 , then (a) is simply a redundant condition. To avoid this redundancy it is plausible to distinguish the two references to benefits this way: that a benefit follows or is caused by an intervention is one condition, that a risk is justified in relation to some benefit is another. The first is a causal criterion, requiring that the subjects are expected to receive a benefit; the second reference to benefit is strictly an ethical one. To adjudicate whether a study satisfies (a), an IRB must look to the magnitude and duration of the benefits subjects are expected to experience and "see" whether they justify the risks. More importantly, I think that the reference to "anticipated benefits" needs to consider those benefits other than the "direct benefits" mentioned as a condition for applying 405 .

To motivate why I think these benefits are different, consider an example from Friedman et al. Now consider a variation on the cancer study in which the biopsy still does not provide any information that is relevant to the subjects' clinical care, but it will provide information that is necessary for a valid assessment of the experimental medication, and there is no less risky way to obtain this information. For example, the biopsy might be needed to assess whether the medication is penetrating the tumor ${ }^{17}$.

Friedman et al. note that the biopsy itself poses risks to the subjects, but does not offer a direct benefit - we may presume only that the study intervention itself offers the direct benefit. So, whereas the intervention causes $\mathrm{X}$ (where $\mathrm{X}$ is some diminution in or cure of the disease or pathology being addressed), the test to know whether the intervention causes $\mathrm{X}$ does not offer a direct benefit. In response

12. Charles Weijer. 2004. "The Ethical Analysis of Risk in Intensive Care Unit Research," Critical Care 8: 85-86.

13. M.J. Field and R.E. Behrman, eds., Ethical Conduct of Clinical Research Involving Children (Washington D.C.: National Academies Press, 2004 ), 138.

14. Friedman et al. "Which Benefits of Research Participation Count as 'Direct'?” p. 6.

15. See Eleonore Stump, Wandering in Darkness: Narrative and the Problem of Suffering (New York: Oxford University Press, 2010 ), 378.

16. Emanuel, Ezekiel J.; Wendler, David; and Grady, Christine. 2000. “What Makes Clinical Research Ethical?” JAMA 283(20): 2701-11.

17. Friedman et al. "Which Benefits of Research Participation Count as 'Direct'?" p. 6. 
to this example Freidman et al. prefer to redefine direct benefit more broadly to include scientifically necessary activities, those activities that are necessary to discern whether the research intervention works. I prefer to take the approach that direct is still a straightforward causal notion, and "anticipated benefit" is the broader notion. As I see it, their example can be handled in the following way: the concept of anticipated benefit cannot be reduced to a physiological effect in the subjects alone. Essentially constitutive of the research enterprise is to come to know things. Knowledge that X occurs must be included as an anticipated benefit. Consider doing the intervention without the biopsy to test efficacy. With no procedures outlined in the protocol for knowing whether the intervention worked, no IRB would approve the study. Such a study would present uncompensated risks to subjects in the form of no known benefit. The same ethical reasoning for why the design of a study has to be proper applies here. If the study design is flawed, no reliable conclusions can be inferred from the interventions and whatever risks they present would be for naught. The benefit of knowledge, therefore, is constitutive of what justifies a research study. Assuming component analysis, then, the risks of the test are compensated for by the necessity of the test to obtain knowledge. And the phrase "in relation to" blocks considering benefits that have nothing to do with procuring knowledge specific to the study ends, such as payments for participation or added research procedures that are not connected to the goals of the particular study ${ }^{18}$.

\section{The core argument: pediatric subjects are not afforded greater protection}

With these key phrases understood, I can now turn to the heart of my argument. To see that there is no further protection afforded children; consider the following quotation from section 46.111 which outlines criteria adult research must satisfy:

(a) In order to approve research covered by this policy the IRB shall determine that all of the following requirements are satisfied ${ }^{19}$ :

(2) Risks to subjects are reasonable in relation to anticipated benefits, if any, to subjects, and the importance of the knowledge that may reasonably be expected to result. In evaluating risks and benefits, the IRB should consider only those risks and benefits that may result from the research (as distinguished from risks and benefits of therapies subjects would receive even if not participating in the research).

Notice that even for adult studies the risks must be justified in relation to the anticipated benefits - just as it is in 405 . But if we start from the assumption that pediatric subjects need further protection than what is afforded adult subjects, and section 405 does not provide further protection, then an ethical lacuna exists in the Federal regulations governing pediatric research. Both sections of the regulations here require that the risks be balanced against the anticipated benefits.

But what about the "direct benefit" requirement for pediatric research? If, as I have articulated above, direct benefit and anticipated benefit are to be understood as being conceptually different, is not the requirement that there be a direct benefit for pediatric research a way of providing greater protection than that afforded adults (insofar as 46.111 does not explicitly include reference to a direct benefit)? I think the answer lies in understanding the fundamental moral reason for why research avoids using persons merely as a means; namely, why component analysis plus the proportionality restriction apply to all research.

Assume that an adult research project presents risks to subjects but no direct benefit. Let's assume those risks are bodily harm in the form of toxic effects of an intervention. If there is no direct benefit, then there is no benefit caused by the intervention. But given the proportionality restriction, each risk needs to be compensated for, and given component analysis, each risk needs to be compensated by a benefit of the same sort, namely bodily/health. (One can derive the same conclusion assuming only component analysis which requires that each risk is offset, and borrowing on our intuitions informing why payment is not a benefit for bodily risk, we can derive the conclusion that the risk is not offset and therefore is not reasonable in relation to the anticipated benefits.) Since a number of prominent authors working in research ethics accept component analysis, and they appear not to restrict it to pediatric research, it follows that for risks to be "reasonable in relation to anticipated benefits" (46.111(b)) more than minimal risks for adult subjects must be compensated for by benefits of the same kind.

But this conclusion entails that even for adult research, a direct benefit is required. Is not this an unnecessarily strict requirement? Although the conclusion appears rather strict, I do not think it is at all unnecessary. Assume that a study on adult subjects presents more than minimal risk to subjects. Assume also that the study interventions are non-therapeutic promising only scientific knowledge (which may or may not affect medical practice for future patients in the same subject class). The key moral question we should ask is not just whether subjects can give informed consent, but also whether it is permissible to subject someone to uncompensated harm for the possible benefit of another. Again, let's return to the analogy I am drawing with the philosophy of religion and the problem of evil. Eleonore Stump notes a general principle of justice namely, "Undeserved suffering which is uncompensated seems clearly unjust; but so does suffering compensated only by benefits to someone other than the sufferer"20. She then gives the example of the U.S. military's LSD experiments on soldiers. Assuming this study was well designed and promised to deliver knowledge worth having, we still think that it was a violation of justice. And she notes that at least part of an explanation for this injustice is "a consequence of the fact that the end aimed at did not directly or primarily benefit those who suffered to achieve it" ${ }^{21}$. It is important to note that this explanation functions as an independent reason for the injustice. Assume that the soldiers consent to high doses of LSD, and you still have a reason for thinking that the study is unjust, namely, the benefits, if any, do not accrue to those suffering the harms.

The idea that consent cannot provide a sufficient reason for the permissibility of a study can be shown by another example. Modifying another example from Stump, ${ }^{22}$ consider a large chemical corporation

18. For an analysis of the other key terms and phrases in sub-part D see Dyson and Parsi. 2010. "Characterizing the Level of Risk in Pediatric Research: An Ethical Examination of the Federal Regulations," Journal of Clinical Ethics 21 (3): 212-220, and M.J. Field and R.E. Behrman, eds., Ethical Conduct of Clinical Research Involving Children (Washington D.C.: National Academies Press, 2004). I would like to thank an anonymous reviewer for bringing to my attention the former article.

19. The other requirements pertain to scientific design, equitable selection of subjects, informed consent issues, and safety monitoring.

20. Eleonore Stump, 1986. "Providence and the Problem of Evil," Christian Philosophy ed. Thomas Flint (Notre Dame, IN: University of Notre Dame Press), 51-91, at 66.

21. Ibid, 66

22. Ibid, 66 . 
that aims to test a new technology for cleaning up toxic chemical spills. It plans to spill chemicals into a particularly poor part of India, and then release their "clean-up chemical". They then follow-up residents of the area testing for toxic effects secondary to consuming the drinking water in the spill area. They promise to compensate every injured resident 1000USD (a small fortune for such residents); in return the residents indemnify the company against any future compensation. The townspeople consent to the spill considering the money as sufficient compensation. It appears that even here, an injustice would be committed against the residents, even in the setting of consent, and in spite of what they view as sufficient compensation. Uncompensated harm stands by itself as a reason against the study. If consent procedures were adequate and ensured understanding, then the study would meet that ethical requirement; but of course, it would fail the requirement not to cause uncompensated suffering or harm.

But may not consent on balance provide a reason for such a study's permissibility? I do not see how this can be the case especially in the example of the Indian residents. Examples abound where consent to being treated unjustly simply does not change the act from one of unjust treatment to just treatment. One way to appreciate this point is that in research ethics we are primarily concerned with the moral quality of the researcher's actions, not the subjects'. The researcher's actions remain unjust in this and other examples whether or not subjects consent to being treated as such. The conclusion to draw is that although the U.S. regulations governing research on competent adults do not explicitly mention that there must be a direct benefit, what it plausibly means for risks to be "reasonable in relation to" benefits is that any harm caused by the research intervention must be compensated for by a proportionate benefit.

Although I think the reasoning so far is sound, it delivers the wrong conclusions at least in relation to actual practice. There are several select counter-examples to the conclusions reached so far; those conclusions being that both pediatric and adult research must involve a direct benefit. Putative counterexamples are not hard to find: (a) placebo controlled trials (PCTs) - since the placebo group does not receive a direct benefit, and (b) deliberate introduction of air pollutants into healthy subjects, ${ }^{23}$ or phase 1 toxicity studies on healthy adult subjects. $^{24}$

The response to PCTs is that although the placebo group does not receive the research intervention, it is precisely because of this that they also are not exposed to the risks of such an intervention. The only source of harm for the subjects in the control group of a PCT is the effect of being without a potentially effective therapy. But if equipoise is satisfied, namely, if it is not known whether administering the intervention or withholding it is better or worse, then there is no reason to favor one over the other. The control group is not being deprived of something effective as far as the researchers know.

Counterexamples falling into group (b) are harder to obviate. Contemporary research practice utilizes healthy adult subjects and many interventions subject these adults to risks without any compensating direct benefit. If asked why they consent to enter such research, the typical reply is for the money. ${ }^{25} \mathrm{I}$ wish to challenge this putative counter-example by challenging the underlying assumption that such studies are permissible in the first place. Most of the research in this class (risks to healthy subjects with no promise of benefit either directly to them or to other persons in their "class," namely, healthy persons) is ethically questionable. If the study by Almeida et al. (note 24 ) is correct, we can begin by imagining such research wherein the protocol does not offer payment to subjects. Considered without payment it becomes apparent that no one would consent to such research - leaving everything the same as it would be with payment. ${ }^{26}$ That no one would consent to such research is a backhanded indicator of where the research stands from a rational choice perspective. It is simply not reasonable to enter such a study considered apart from the payment. Since the study's risks and benefits to which the IRB must weigh preclude considering benefits in the form of payment $\mathrm{s}^{27}$ the riskbenefit ratio of the study is what provides the chief ethical justification for it. But if it is inconsistent with rational choice to enter the study sans payment, then the study itself presents risks that are not "reasonable in relation to the anticipated benefits." And therefore, the study appears to fail 46.111(b). The fact that payment must be added to the research protocol to make it an object of one's rational choice manifests how unreasonable the risk-benefit ratio of the research is in itself.

The line of reasoning here suggests that payment cannot be used to justify research that presents otherwise uncompensated risk to healthy adult subjects. But this reasoning is easily duplicated in the case of pediatric research. So here again, there appears to be shared ethical reasoning justifying research for these two different populations. If the same reasoning applies, then children are not afforded greater protection than that afforded adults. It is obvious that actual practice includes research on healthy adults with no promise of direct benefit. But if the only reason for entering such studies is the offer of payment, such studies are ethically suspect, and "actual practice" cannot function as an ethical justification in this regard. Rather, it is the other way around; sound ethical reasoning should judge actual practice.

So, on some understanding of "direct benefit" all research that presents more than minimal risk (and more than a minor increase over minimal risk) must provide a compensating benefit. This moral requirement flows from the plausible position that subjecting persons to uncompensated harm or suffering for the benefit of another is an independent reason against the permissibility of a study. And although actual practice includes research on healthy subjects with no promise

23. For example, Aris RM, et. al. 1993. "Ozone-induced airway inflammation in human subjects as determined by airway lavage and biopsy," American Review of Respiratory Disease 148(5):1363-72. See also, Balmes JR, et. al. 1997. "Effects of ozone on normal and potentially sensitive human subjects. Part I: Airway inflammation and responsiveness to ozone in normal and asthmatic subjects," Research Report (Health Effects Institute) June (78): 1-37.

24. David B. Resnick and Greg Koski. 2011. "A National Registry for Healthy Volunteers in Phase 1 Clinical Trials,” JAMA March 23, 305(12): 1236-1237.

25. Almeida L, Azevedo B, Nunes T, Vaz-da-Silva M, Soares-da-Silva P. 2007. "Why healthy subjects volunteer for phase I studies and how they perceive their participation?" European Journal of Clinical Pharmacology 63(11):1085-1094.

26. To tether my reflections here to a particular case, I am thinking of the TGN 1412 trial which offered subjects $\sim 3500$ SD. Now consider the study without such a payment offer. It is apparent that no one would consent to it. See Emanuel EJ, Miller FG. 2007. "Money and distorted ethical judgment about research: ethical assessment of the TeGenero TGN1412 trial." American Journal of Bioethics 7: 76-81.

27. The Office of Human Research Protections give the following guidance, "Direct payments or other forms of remuneration offered to potential subjects as an incentive or reward for participation should not be considered a "benefit" to be gained from research.... Although participation in research may be a personally rewarding activity or a humanitarian contribution, these subjective benefits should not enter into the IRB's analysis of benefits and risks." (Emphasis added). See Institutional Review Board Guidebook: Chapter III Basic IRB Review, http://www.hhs.gov/ohrp/irb/irb_chapter3.htm (accessed September 21, 2011). 
of direct benefit, such studies are at best ethically suspect: payments and actual practice do not function as ethical justifications for such research. $^{28}$

Even if this much is granted, however, my argument meets further obstacles. One may object that I have omitted mention of clause (b) in section 405. There it tells us that the risk-benefit profile of the research cannot be worse than the risk-benefit profile of available alternatives alternatives such as not being in the study. And a counterpart for this clause does not show up in section 46.111 governing adult research.

In response, the objection assumes that measuring the risksbenefits of the research against the risks-benefits of alternatives provides further protection of pediatric subjects. But this assumption is suspect. Consider research $\mathrm{R}$, where the risk-benefit profile favors the risks. Assume next that the alternative also has a risk-benefit profile which heavily favors the risks. In such a scenario, the IRB may consider - consistent with 405 - that $\mathrm{R}$ is permissible in that (i) the risks are justified by the anticipated benefits and this is so partly because (ii) the alternatives are just as bad. Such a justification is not available to the IRB considering adult studies in that section 111 specifically prohibits comparing the risk-benefit profile of the research with other alternatives. 46.111 (2) says, "In evaluating risks and benefits, the IRB should consider only those risks and benefits that may result from the research (as distinguished from risks and benefits of therapies subjects would receive even if not participating in the research)." ${ }^{29}$ If $\mathrm{R}$ were recruiting adults, the IRB would assess the risk-benefit profile of $\mathrm{R}$ on its own, and would probably not approve it. Adult research stands or falls on its own merits, whereas pediatric research may, as in $\mathrm{R}$, be brought to life via appeal to alternatives. Thus, though appeal to alternatives may provide further protections for pediatrics, such an appeal is a double-edged sword. As such, section 405 as a whole provides no further protection to pediatric subjects than for adult subjects.

Now consider a more refined reply according to which one considers the alternatives of not being in the research at all. There are at least three scenarios to consider here:

The risk-benefit profile of not being in the study is less favorable than the risk-benefit profile of being in the study. Under these circumstances one would be hard pressed to say that the research under review is impermissible. But it is questionable how common this scenario would be. The difference maker between these two risk-benefit profiles is the research intervention; that is what appears to make the risk-benefit profile favor the research. But if it is really known that the research risk-benefit profile is more favorable, then why do the research? Why is the study one of procuring knowledge? This reveals an important epistemic point about justifying research on vulnerable populations. If the research is really research, then one is in a state of aporia as to whether the research intervention will be effective. Furthermore, the research intervention is being tested against alternatives. To draw any valid conclusions from the evidence, the risk-benefit profiles of the alternatives must be known going into the research. There has to be a fixed comparison point from which to draw empirically informed conclusions sufficient to change medical practice. This means that to evaluate the research risk-benefit profile as being better, one must know it to be so with as much certainty as the alternatives. Being in such an epistemic position is typically not the case in the research setting.

- The risk-benefit profile of not being in the study is more favorable than the risk-benefit profile of being in the study. Here, it seems clear that the research in question is not justifiable.

The risk-benefit profiles are in equipoise and that is why the research is being done. This is probably the case with most research, there is a state of aporia as to which is best medical practice for addressing a particular disease or pathology. But notice, in the setting of equipoise, there is no reason for a study being permissible. That an alternative is equivalent to being in the study cannot be used as a justification for the permissibility of the study. There is, however, an obvious scientific justification, namely, to find out if the study is in fact better than alternatives. But recall from the syllabus of ethical errors in research, the knowledge gained cannot ethically justify the study; it clearly is not an explanation for why the vulnerable subjects are receiving further protection. Gaining knowledge is not itself a justification that subjects will receive further protection from research harms.

Pointing out an ethical lacuna in the regulations does not entail that the regulations are bad or wholly unusable. I happen to think that the U.S. regulations, the International Conference on Harmonization Guidelines on Good Clinical Practice, and the Declaration of Helsinki, provide sufficient ethical guidance where such guidance is needed. The take home message of this paper is not so much to change or add to the regulations governing children, but for research review boards to take note of the ethical lacuna adumbrated here, and to be aware that applying section 405 to a research protocol may not be morally sufficient. IRB's should constantly ask themselves, when reviewing pediatric research, whether the protections in place are more than (understood qualitatively or quantitatively) those we would expect for adult subjects.

Of course, taking this attitude to pediatric research may have the result that more studies get disapproved, (specifically, those studies that would otherwise get approved without taking this attitude of further protection). Robert Rosenfield decries such a result. ${ }^{30} \mathrm{He}$ opens his article with the following comment,

In spite of a mandate for the inclusion of children in federally funded research, the current regulatory environment, designed to protect children, imposes barriers to research in children that are a disincentive to high-quality clinical research in minors. ${ }^{31}$

What follows is a recapitulation of his own experience regarding the review of his own study on polycystic ovarian syndrome (PCOS). His study was reviewed under the mechanism for studies in category 45.407. Category 407 is reserved for research that is otherwise not approvable under the previous three categories; 404 which pertains to research satisfying minimal risk; 405 , noted above, and 406 which pertains to research that is more than minimal, and does not hold out a

28. The importance of the knowledge gained could justify such research, but this is also the case for pediatric research as outlined in section 406 of Sub-part D. Again, if the same ethical reasoning applies to both pediatric and adult research, then children are not afforded greater protection.

29. Emphasis mine.

30. Robert L. Rosenfield. 2008. "Improving Balance in Regulatory Oversight of Research in Children and Adolescents: A Clinical Investigator's Perspective," Annals of the New York Academy of Medicine 1135: 287-295. (Emphasis mine).

31. Ibid, 287. 
prospect of direct benefit for the subjects, but represents only a "minor increase" over minimal risk. The focus of Rosenfield's complaints, however, has nothing to do with the regulations themselves, nor with their ethical importance. He notes, "[t]his institutional process was a study in bureaucratic nitpicking: there were more than 20 iterations by the time the process was complete 3 years later." ${ }^{\prime 2}$ There is nothing in this or in the other complaints that challenge the ethical role SubPart D plays in protecting pediatric subjects. How individual IRB's apply the regulations and their own standard operating procedures is a separate issue and one that is more appropriately classified as an organizational ethics issue than one squarely within research ethics. And it appears that Rosenfield's complaints are organizational in nature. Furthermore, Rosenfield recapitulates his experience with the review process governing research in category 407. By his own admission, this review process is exceedingly rare, nine reviews have been conducted since 407's inception in $2002,{ }^{33}$ and it involves multiple agencies. But this begs the question, why should such research undergo such strict review? Rosenfield appears to think it is obvious that such oversight is over-the-top. "Research in healthy children that exceeds the regulatory definition of minimal risk cannot be approved by an IRB. ${ }^{34}$ But why should it be the case that exposing healthy children to more than minimal risk per research intervention should not receive greater oversight? To this question Rosenfield is silent.

In summary, I have addressed an ethical lacuna in the U.S. regulations aimed to protect pediatric subjects. In particular, research satisfying CFR 46.405 may not be morally sufficient to permit a study. This conclusion follows if we think that pediatric subjects are entitled to greater protection than adults subjects. Comparing 405 with 46.111, I argued that there is no further protection afforded pediatric subjects beyond what is granted adults. The take home message, however, is not to further refine the regulations, but for IRBs to be aware of this lacuna. Ethical deliberation is not reducible to applying an algorithm like solving a mathematical equation may be. Ethical thinking has an element of individual creativity and art to it. Ultimately, our ethical decisions flow from our consciences and our consciences are perfected through growing sensitivity to ethically fine details of a situation and, in the case at hand, a research protocol. ${ }^{35,36}$

\section{References}

1. There are now a series of meetings, in particular Meeting Five, being conducted by the President's Council on Bioethics aimed to address this issue.

2. Richard E. Behrman, "Preface," Ethical Conduct...

3. Department of Health and Human Services. Code of Federal Regulations. Title 45, Part 46, Protection of Human Subjects, subpart D Available at: http://www. hhs.gov/ohrp/humansubjects/guidance/45cfr46.html [accessed September 21 2011].

4. Dyson and Parsi

5. King NM (2000) Defining and Describing Benefit Appropriately in Clinical Trials Journal of Law Medicine and Ethics 28: 332-343. 